\title{
BRANCHED EXTENSIONS OF CURVES IN ORIENTABLE SURFACES
}

\author{
BY
}

\author{
CLOYD L. EZELL AND MORRIS L. MARX
}

\begin{abstract}
Given a set of regular curves $f_{1}, \ldots, f_{\rho}$ in an orientable surface $N$, we are concerned with the existence and structure of all sense-preserving maps $F: M \rightarrow N$ where

(a) $M$ is a bordered orientable surface with $\rho$ boundary components $K_{1}, \ldots, K_{\rho}$,

(b) $F \mid K_{i}=f_{i}, i=1, \ldots, \rho$,

(c) at each interior point of $M$, there is an integer $n$ such that $F$ is locally topologically equivalent to the complex map $w=z^{n}$.
\end{abstract}

1. Introduction. A set of curves in a surface (regular maps from the unit circle into the surface) is called normal if the set of intersection points (self-intersections as well as intersections of different curves) is a finite set of transverse double points. We are concerned with the following problem:

Given a normal set of curves $f=\left\{f_{1}, f_{2}, \ldots, f_{\rho}\right\}$ in an orientable surface $N$, find all sense-preserving maps $F: M \rightarrow N$ where

(a) $M$ is a bordered orientable surface with $\rho$ boundary components $K_{1}, \ldots, K_{\rho}$.

(b) At each interior point of $M$ there is an integer $n>1$ such that $F$ is topologically equivalent to the complex map $z^{n}$. A point where $n>1$ is called a critical point of multiplicity $n-1$; the image of a critical point is called a branch point.

(c) $F$ is locally one-to-one on the boundary $\partial M$ of $M$, there are no branch points on the images of curves in $f$ and $F \mid K_{i}=f_{i}$, for each $i$.

Such a map $F$ is called a polymersion extension of the curves $f$. (We assume here that the orientation on $M$ is chosen so that the interior of $M$ is to the left of the boundary curves.)

The problem above, in the case where $N$ is the plane, $f$ a single curve and $M$ a disk, was posed by C. Loewner about 1948. (Actually, in Loewner's problem, the curve was not necessarily normal and the extension $F$ was to be analytic.) Loewner was interested in what conditions the curve must satisfy, other than possessing nonnegative circulation, to guarantee the existence of such extensions.

Titus [11] gave a solution to Loewner's problem for normal curves by giving an algorithm (involving modification of the given curve by a series of cuts of certain types) which allows one to decide whether or not there exists a polymersion extension. By the uniformization theorem of Koebe, the existence of a polymersion

Received by the editors March 15, 1978 and, in revised form, July 10, 1979. Presented to the Society, January 26, 1977 (St. Louis), and April 7, 1978 (Houston).

AMS (MOS) subject classifications (1970). Primary 55A10; Secondary 05C10, 30A90, 57A35, 57D45. 
extension is equivalent to the existence of an analytic extension, and Loewner's question was answered. However, Titus did not explicitly deal with the problem of finding all of the nonequivalent extensions (the extensions are equivalent if there is a homeomorphism $h: M \rightarrow M^{*}$ with $F h=G$ ) and S. Blank [2] gave a solution to this problem for the case where $F$ is a local homeomorphism. Expanding on Blank's work, Marx [8] defined a combinatorial invariant (called a maximal assemblage) constructed from a given normal curve that shows the existence of extensions, reveals their branch point structure, and enables one to count the number of equivalence classes of extensions. A generalization of the Marx assemblage that determines extensions from arbitrary orientable surfaces mapping to the plane or sphere has been given by Francis in [4].

This brings us to the present problem of defining an assemblage for curves $f$ in arbitrary orientable surfaces. Francis gives a solution to this problem in [5] by taking a map $G$ (called a model map) from the target surface $N$ to the sphere and defining an assemblage for $f$ based upon the assemblage of the projection $G(f)$ in the sphere. Our approach is to extend the Francis definition of the planar (or spherical) assemblage given in [4] to make sense in a surface of arbitrary genus. Thus we get an assemblage, constructible directly from a set of curves in a surface, without the use of a model map. With this scheme we obtain

(i) higher genus analogues of the existence theorems in [4] for the plane and sphere (Theorem 2.3),

(ii) a generalization of the winding number of a planar curve to curves in surfaces of higher genus and some applications (Theorem 3.2 and Corollary 3.3),

(iii) a theorem on the existence of polymersion extensions that generalizes the main result of [9] (Theorem 3.4),

(iv) a solution to what should be called the Loewner-Titus conjecture [12, p. 218] for curves on an orientable surface of genus $>0$ (Corollary 3.5),

(v) a Riemann-Hurwitz type formula (Theorem 3.7).

To help the reader understand the origin of the tools developed here, we have, for the most part, produced sufficient conditions for the existence of extensions by assuming the existence of the desired polymersion and then defining necessary conditions that turn out to be sufficient.

2. Polymersions and assemblages. Let $F: M \rightarrow N$ be a polymersion extension of some normal mapping $f: K \rightarrow N$. Select a point $\infty \in N-f(K)$ which is not a branch point of $F$. The set of curves $f(K)=[f]$ may be decomposed into a set of nonoverlapping simple closed curves, called Gaussian circles, by separating and smoothing at the intersection points. See Figure 1 below.

A simple closed curve that bounds a disk not containing $\infty$ is positive (negative) if the disk lies to the left (right) of the curve relative to the outwardly pointing normal vectors on $N$. In Figure 1, the curve $[f]$ produces three Gaussian circles, $g_{1}$, $g_{2}$ and $g_{3}$. Circle $g_{1}$ is negative and $g_{2}$ positive. Circle $g_{3}$ does not bound a disk.

Let $\left[f_{i}\right], i=1, \ldots, \rho$, be the components of $[f]\left(\right.$ i.e. $\left.\left[f_{i}\right]=f\left(K_{i}\right)\right)$. Each curve $\left[f_{i}\right]$ has a tangent winding number, twn, relative to any vector field on $N$ whose zeroes do not occur on $\left[f_{i}\right]$. The tangent winding number is defined as the number of 
times a vector tangent to the curve rotates relative to the vector field as the curve is traversed one time. (A counterclockwise rotation is considered positive.) See [3]. The tangent winding number of $[f]$ is defined to be the algebraic sum of the tangent winding numbers of its components. Note that the usual definition of twn of a curve in a plane corresponds to the above, relative to a field of parallel vectors.

It follows directly from the definitions above that $\operatorname{twn}[f]$ equals the sum of the tangent winding numbers of its Gaussian circles. It follows from [3, Lemma 2.6], that the twn of a positive (negative) Gaussian circle bounding a disk with no zeroes is $+1(-1)$. Hence if $[f]$ is contained in a disk in $N-\{\infty\}$ with no zeroes, $\operatorname{twn}[f]$ equals the number of positive Gaussian circles minus the number of negative ones and is independent of the particular choice of the vector field.

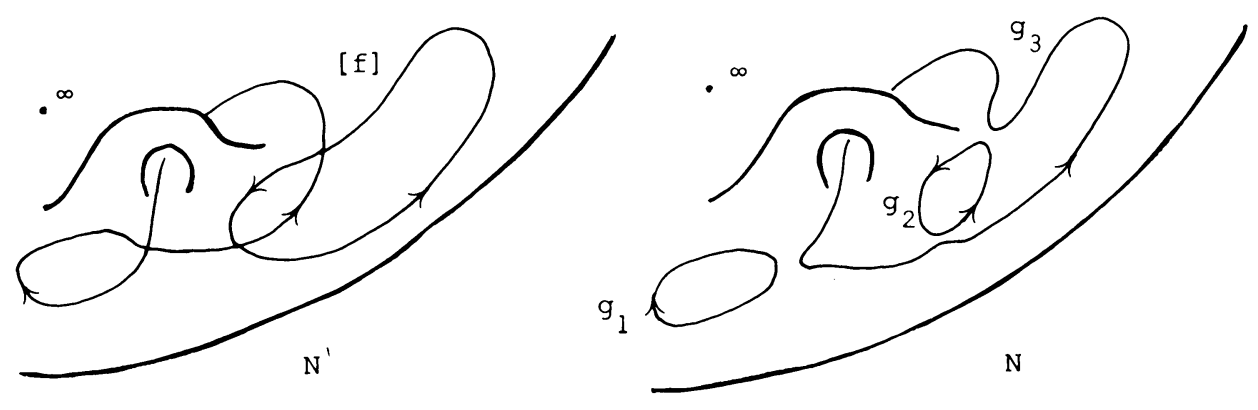

Figure 1

We shall now describe a set of data, called the induced assemblage on the curves [ $f$ ], defining a cellular decomposition of $M$ on each cell of which $F$ is univalent. The assemblage determines the cell boundaries and the identification scheme, hence, $F$ can be reconstructed (up to topological equivalence) from the assemblage. The assemblage $A_{F}=(R, W, S, P)$ will consist of a set $R$ whose members are called rays, a possibly empty set $W$ whose members are called wheels, and two permutations $S$ and $P$ called, respectively, the successor and assembling permutations. A description of these objects is below.

Choose a vector field on $N$ vanishing only at a finite number of points in a disk neighborhood $U \subset N-[f]$ of $\infty$. (Such vector fields are known to exist. See [6, p. 146, Exercise 12].) Denote the cardinality of $F^{-1}(\infty)$ by $\beta$. Choose $\beta$ concentric circles $w_{1}, \ldots, w_{\beta}$, each of which lies entirely in $(N-[f]) \cap F(M)$ and each bounding a disk containing $U$ but no branch points. Orient these circles so that the disks they bound lie to their right.

Now select a set of oriented, simple closed curves, $r_{1}, \ldots, r_{k}$, that are representatives for a minimal generating set of the fundamental group $\pi(N, \infty)$ of $N$ based at $\infty$. For each branch point $x \in N$ select an oriented arc initiating at $x$ and terminating at $\infty$, producing a set $r_{k+1}, \ldots, r_{j}$. Finally, add (if necessary) oriented arcs $r_{j+1}, \ldots, r_{m}$, to insure that $R$ is sufficient, i.e., there is a ray originating inside of every negative Gaussian circle, and every component curve in $f$ is crossed by 
some ray. (If there is at least one ray emanating from each component of $N-[f]$, we call $R$ full.) Then define $R$ to be $\left\{r_{1}, \ldots, r_{m}\right\}$. We assume each ray is chosen so that it intersects the curves $f \cup W$ transversely, avoids the intersection points of $f$, and does not intersect any other ray. The intersection points of $f \cup W$ and $R$ are called crossings and we say a crossing is positive (negative) if the curve crosses the ray from right to left (left to right). We will use the letter $\nu$ for the number of negative crossings.

We define simultaneously the permutation $P$ and a graph $G_{A}$ imbedded in $M$. (We will see that the imbedding of $G_{A}$ in $M, G_{A}(M)$, gives the cellular decomposition of $M$ mentioned above.) If $F^{-1}(\infty)$ is not empty, select any one-one correspondence between $F^{-1}(\infty)$ and $W$. For each point $\infty \in F^{-1}(\infty)$, let $\bar{w}$ be the inverse image of the wheel corresponding to $\bar{\infty}$. Let $\bar{W}=\left\{\bar{w}_{1}, \ldots, \bar{w}_{\beta}\right\}$. For vertices of $G_{A}$ take the preimages of the branch points in $N$ and all preimages of crossings which lie on $\partial M \cup \bar{W}$. Include as edges all arcs on $\partial M \cup \bar{W}$ joining the vertices and additional arcs chosen as described below. Let $x$ be a negative crossing on a ray $r$ with boundary preimage $\bar{x}$. Lift $r$ from $\bar{x}$ until it terminates at some point $\bar{y}$ on $\partial M \cup \bar{W}$. Note that $f(\bar{y})=y$ is a positive crossing. Include the arc $[\bar{x}, \bar{y}]$ as an edge in $G_{A}$ and let $(x y)$ be a cycle of $P$. Now let $\bar{b}$ be a preimage of some branch point $b$ that is the base of some ray $r$. If the multiplicity of $\bar{b}$ is $m-1$, then there are $m$ arcs $\left[\bar{b}, \bar{z}_{1}\right], \ldots,\left[\bar{b}, \bar{z}_{m}\right]$ emanating from $\bar{b}$, each covering a segment of $r$, and terminating on $\partial M \cup \bar{W}$ at preimages $\bar{z}_{1}, \ldots, \bar{z}_{m}$ of positive crossings $z_{1}, \ldots, z_{m}$. Include these arcs as edges of $G_{A}$ and let $\left(z_{1}, \ldots, z_{m}\right)$ be a cycle of $P$, where the order is opposite that determined by the orientation on $M$ at $\bar{b}$. Note that any crossing not in a fan has preimage lying on a pendant edge and is a fixed point of the permutation $P$ on the crossing set. Note that the nontrivial cycles of $P$ are either pairs (transpositions with one negative and one positive crossing) or fans (cycles with only positive crossings from the same ray). The number of crossings contained in the fans minus the number of fans is called the partial branching number $\pi$.

Finally, we define the permutation $S$ on the set of all crossings as the map which takes a crossing on a component curve (an $f_{i}$ or $w_{i}$ ) to the next crossing on that curve in the direction of its orientation.

The closed curves (other than wheels) in [ $f \cup W]$ that are the images under $F$ of the oriented face boundaries of $G_{A}(M)$ are called the orbits of the assemblage. An orbit is not necessarily regular or normal but can be approximated arbitrarily closely by a regular, normal curve which does not intersect any ray (these approximations were called modifications in the previous literature). Hence, we can define the tangent winding number of an orbit by taking it to be the tangent winding number of a "sufficiently close" regular approximation. Now it follows from the definition of $S$ and $P$ that the orbits are readily located using these permutations as described below. Pick any crossing $x$ and follow the curve from $x$ to the next crossing $y=x S$. If $y$ belongs to a pair, proceed from $y$ on the ray containing $y$ to $y P=x S P$. If $y$ is part of a fan, follow the ray containing $y$ to its base and then proceed back up the ray to $y P=x S P$. Repeat the process by applying $S P$ to the new crossing $x S P$. Continue the path in this manner until it 
terminates at the starting point $x$. The closed curves of $[f \cup W]$ found this way are the orbits and the number of orbits equals the number $\zeta$ of cycles in the permutation $S P$.
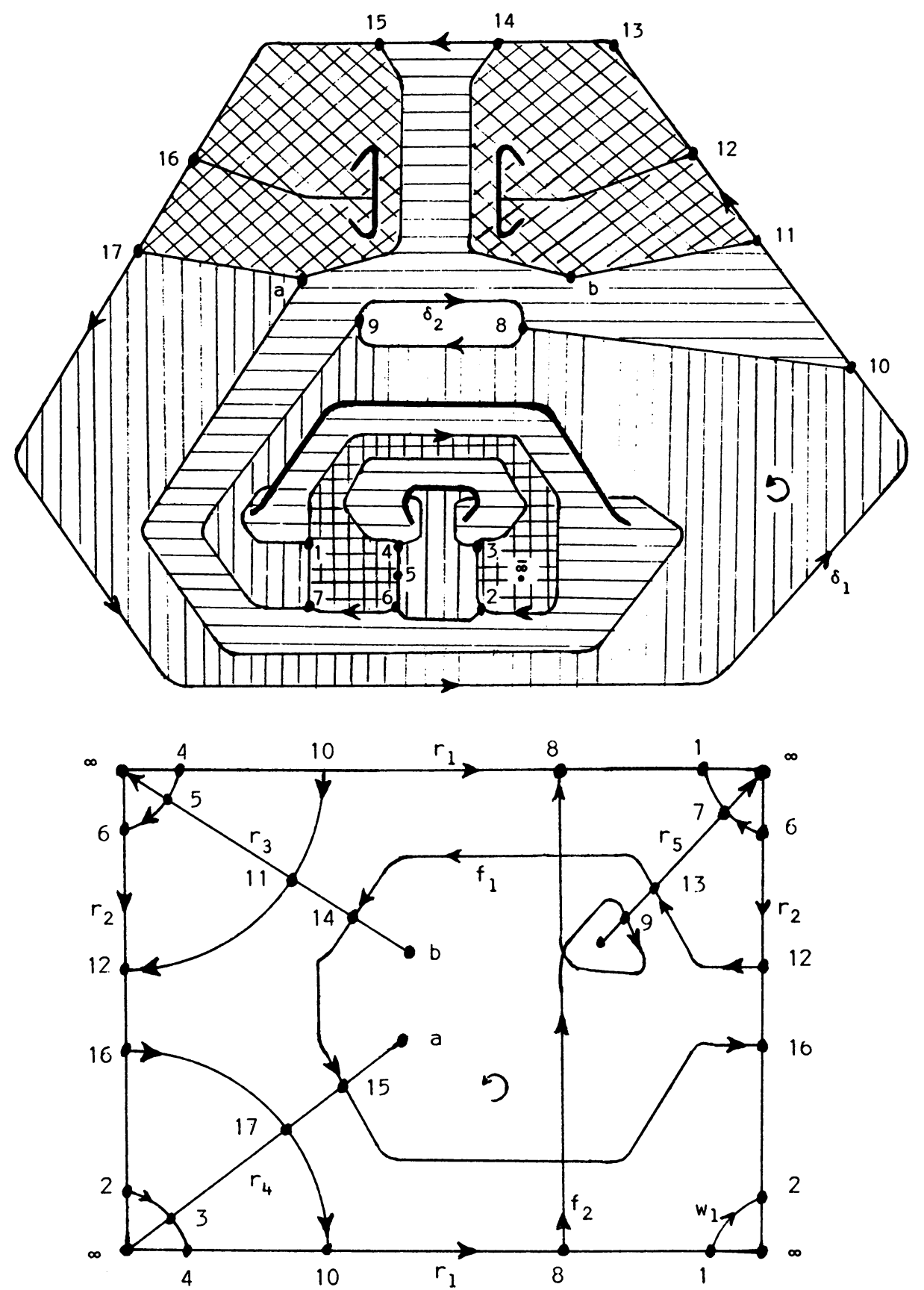

FIGURE 2 
The objects described above are illustrated in the following example.

2.1. ExAMPLE. This example illustrates an assemblage induced by a polymersion mapping a two-holed torus with two boundary components to a torus. The domain and target spaces are shown in Figure 2. The boundary components are $\delta_{1}$ and $\delta_{2}$ in the domain and their images are the curves $\left[f_{1}\right]$ and $\left[f_{2}\right]$ in the target. There are five rays in the ray set $R=\left\{r_{1}, r_{2}, r_{3}, r_{4}, r_{5}\right\}$ and one wheel in $W=\left\{w_{1}\right\}$. The single preimage of $\infty$ is $\bar{\infty}$. The successor permutation $S$ and assembling permutation $P$ are below:

$$
\begin{aligned}
& S=\left(\begin{array}{llll}
1 & 234567)(89)(1011121314151617
\end{array}\right), \\
& P=(108)(41)(1216)(62)(97)(1411)(15317)(13)(5) \text {. }
\end{aligned}
$$

The first five cycles in $P$ are pairs and the remaining four are fans.

This assemblage has three orbits shown below:

$$
\begin{aligned}
& O_{1}=(12,13,14, b, 11,12,16,17, a, 15,16,12), \\
& O_{2}=(8,9,7,1,4,5,6,2,3, a, 17,10,8), \\
& O_{3}=(1,2,6,7,9,8,10,11, b, 14,15, a, 3,4,1) .
\end{aligned}
$$

Note that the orbits correspond to the cycles in $S P$,

$$
S P=\left(\begin{array}{ll}
1213111615)(8745217)(69101431)
\end{array}\right)
$$

The regions bounded by the orbits are shown in Figure 3 and are shaded to correspond to their preimages in the domain. The checked region in the domain containing the point $\infty$ is the preimage of the disk bounded by the wheel.

Recall, our program is to identify those necessary properties of induced assemblages that turn out to be sufficient for constructing polymersion extensions. These are listed below, with brief proofs where needed.

(1) In every pair of the assembling permutation $P$, the negative crossing precedes the positive on the ray containing both. (Such a pair is called a positive pair. A pair with the positive crossing preceding the negative will be called a negative pair.) An assembling permutation with this property is called effective; an assemblage is effective when it has an effective assembling permutation.

(2) The number of negative crossings of $f$ on any generator ray $r$ equals the number of positive crossings on $r$. We call this property of $f$ the generator parity condition, GPC.

For each negative crossing $x$ on $r$, lift the loop $r$ from the preimage of $x$ on $\partial M$. If it terminates at a point on $\partial M$, stop. Otherwise it terminates at a point of $F^{-1}(\infty)$. From this point lift $r$. Continue in this manner until the termination is at a point of $\partial M$, which must be a positive crossing. Repeat for each negative crossing on $r$. Since $r$ does not contain any branch points, we have defined a one-one correspondence from the negative crossings on $r$ to the positive. Now repeat this lifting argument beginning with the positive crossings to obtain a one-one correspondence from the positives to the negatives. Note we have shown that a set of curves that are the boundary images of a polymersion satisfy GPC for an arbitrary choice of $\infty$ and generator rays. 
BRANCHED EXTENSIONS OF CURVES
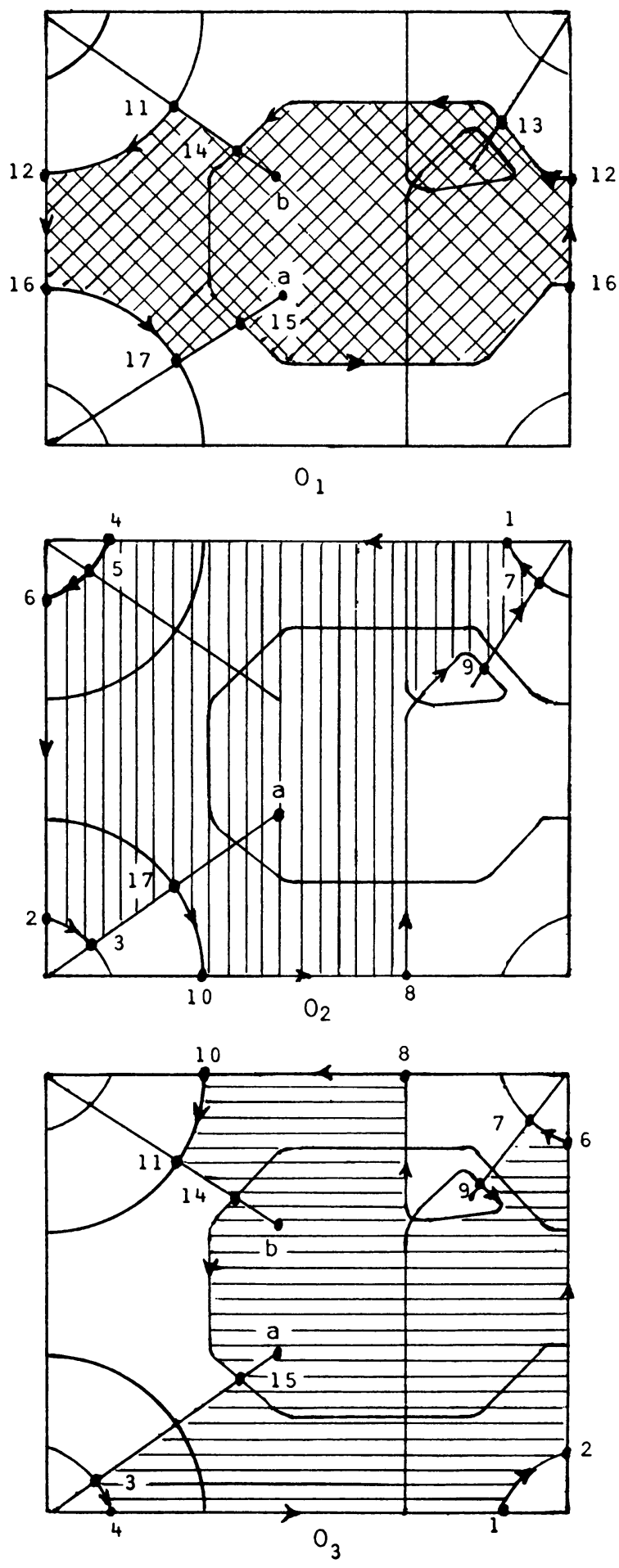

FiguRE 3 
(3) The permutations $S$ and $P$ generate a transitive group; this follows easily from the fact that the domain space $M$ is connected. In such a case we say the assemblage is transitive.

(4) Each orbit of the assemblage is a positively turning (i.e., possess no negative Gaussian circles) closed curve contained in a disk in $N$.

The orbits are contained in disks since, by definition of $P$, the orbits do not cross the generators of the fundamental group. The fact that they are positively turning follows from the effectiveness of $P$ and the sufficiency of the raying.

(5) The imbedding $G_{A}(M)$ is cellular.

To see this, take $M^{*}$, a face (with boundary) in the imbedding $G_{A}(M)$, and show it is a disk. Since $F\left(\partial M^{*}\right)$ is an orbit (or set of orbits) of $A$, we can by (4) and a sufficient smoothing of $F\left(\partial M^{*}\right)$, obtain an immersion $F^{*}$ from $M^{*}$ to $N$ with $F^{*} \mid \partial M^{*}$ a set of positively turning regular curves all lying in a disk in $N$. Compose $F^{*}$ with a sense-preserving homeomorphism from the disk in $N$ containing $F^{*}\left(M^{*}\right)$ to the plane to obtain an immersion $F^{* *}$ of $M^{*}$ to the plane with $\partial M^{*}$ mapping to a set of positively turning regular curves $\left[f^{*}\right]$ in the plane.

It is a known result (the Riemann-Hurwitz formula for polymersions into the plane) that if $F: M \rightarrow R^{2}$ is a polymersion from a compact bordered surface to the plane with $F(\partial M)=[f]$, then $\chi(M)=\operatorname{twn}[f]-\mu(F)$, where $\mu(F)$ is the total multiplicity of $F$. Since $F^{* *}$ is an immersion, $\mu\left(F^{* *}\right)=0$ and we have $\chi\left(M^{*}\right)=$ $\operatorname{twn}\left[f^{*}\right]$. But $\operatorname{twn}\left[f^{*}\right]$ must be positive. The only possibility is that $M^{*}$ is a disk and $\left[f^{*}\right]$ is a simple closed curve.

(6) Each orbit can be approximated by a positively oriented simple closed curve contained in a disk not containing $\infty$.

This is a corollary of (5). These curves cannot bound $\infty$ since an orbit is the image of a face in $G_{A}(M)$ containing no preimage of $\infty$. We indicate this property of an orbit by saying the orbit is simple. To say an assemblage is simple means all of its orbits are simple.

Note. Thus far we have observed that induced assemblages are effective, transitive and simple.

(7) The multiplicity $\mu$ of $F$ equals the partial branching number $\pi$ of the assemblage.

This follows from the fact that each fan of length $m$ corresponds to a critical point of multiplicity $m-1$.

(8) $\chi(M)=\zeta-\nu-\pi+\beta$.

This is calculated from the Euler formula by observing the following: the number of faces in $G_{A}(M)=\zeta+\beta$; the number of edges in $G_{A}=\pi+c+\nu+n$ where $c$ is the number of preimages of critical points and $n$ is the number of crossings on $f \cup W$; and the number of vertices in $G_{A}=n+c$.

(9) The polymersion $F$ can be reconstructed up to a homeomorphism of $M$ from the assemblage. As a corollary to this, if $F$ and $G$ have the same induced assemblages they are equivalent.

Since the assemblage $A$ on $F(\partial M)$ determines a cellular decomposition of $M$ on each cell of which $F$ is univalent, and since this cellular decomposition can be 
determined by examination of the orbits of the assemblage as discussed earlier, we can reconstruct $F$ from the assemblage as follows. Select one disk for each orbit (with boundary corresponding to the boundary of the orbit) and one disk for each wheel and identify in the obvious way to obtain a homeomorph $M_{A}$ of $M$. Define $F_{A}: M_{A} \rightarrow N$ by mapping each cell corresponding to an orbit homeomorphically to the disk bounded by that orbit. (This can be done since the orbits are simple.) Map each cell corresponding to a wheel homeomorphically to the disk bounded by that wheel. Both $F$ and $F_{A}$ are homeomorphisms on corresponding cells in $M$ and $M_{A}$ and are hence equivalent.

2.2. EXAMPLE. The cells used to reconstruct the domain space for the map in 2.1 are shown below.
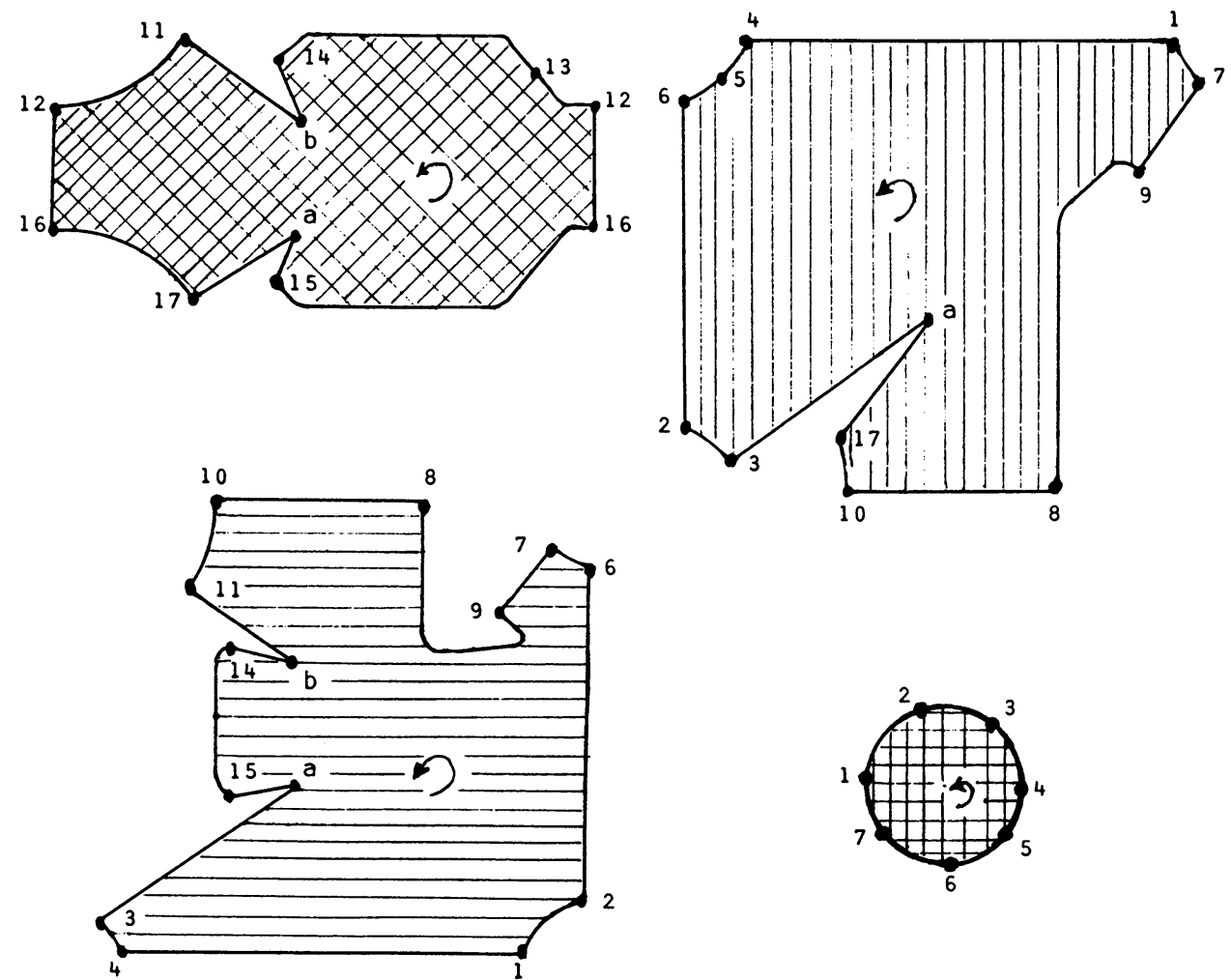

Let $f: K \rightarrow N$ be a normal mapping to an oriented surface $N$. Select $\infty$ and choose any sufficient raying $R$ including the generators of $\pi(N, \infty)$. Let $W$ be a set (possibly empty) of wheels. Define $S$ as before and let $P$ be any permutation on the crossings $(R \cap(f \cup W)$ ) such that if $x$ is a crossing on $r$, then $x P$ is also a crossing on $r$. The set $A_{f}=A=(R, W, S, P)$ is called an assemblage on $f$. The orbits of $A$ are now defined in terms of the cycles of $S P$ as described earlier. (One can check that this definition does indeed determine a set of $\zeta$ closed curves in [ $f \cup W]$ where each segment corresponding to a ray or wheel appears twice.) An assemblage where every negative crossing is paired with a positive crossing is called 
a paired assemblage. As before, an effective assemblage is a paired assemblage with all positive pairs. The definitions of transitive assemblage and simple assemblage are also as before.

The following theorem shows that the necessary conditions discussed in (1)-(9) give the appropriate sufficient conditions for extensions.

2.3. TheOREM. Let $f: K \rightarrow N$ be a normal mapping and let $A$ be a transitive, effective assemblage of $f$ with $\beta$ wheels, $\nu$ negative crossings, $\zeta$ orbits, and with partial branching number $\pi$. Then $f$ has a polymersion extension $F: M \rightarrow N$ from a connected surface $M$ where

$$
\mu(F)=\nu+\operatorname{twn}(f)-\zeta+\pi+\beta(\chi(N)-1)
$$

and

$$
\chi(M)=\zeta-\nu-\pi+\beta .
$$

If, in addition, $A$ is simple, then $A$ identifies (up to equivalence) a particular map $F_{A}$ : $M_{A} \rightarrow N$ with $\mu\left(F_{A}\right)=\pi$ and $\chi\left(M_{A}\right)=\zeta-\nu-\pi+\beta$. If $A$ is an assemblage induced by some polymersion $F$, then $F_{A}$ is equivalent to $F$.

Proof. First, consider the case where $A$ is simple. The map $F_{A}: M_{A} \rightarrow N$ can be constructed as in (9) by building $M_{A}$ with cells corresponding to the orbits and wheels of $A$, and defining $F_{A}$ to be a homeomorphism on each cell. Since $A$ is simple, all branch points occur at the base of rays, and by the construction, the base of every ray containing a nontrivial fan is a branch point. The assembling permutation induced by $F_{A}$ is precisely the permutation $P$ in $A$. It is easily seen as before that $\mu=\pi$ and $\chi\left(M_{A}\right)=\zeta-\nu-\pi+\beta$. If $A$ is induced by $F$, then $F$ and $F_{A}$ are equivalent. This was observation (9).

Moreover, if $A$ is a transitive, paired assemblage that is effective but not simple, one can still construct a normal extension of $f$ as follows. Although the orbits are not simple, it follows from the effectiveness of $P$ and the sufficiency of $R$ that the orbits of $A$ are positively turning closed curves contained in disks in $N$. Construct $M$ as above with one cell for each orbit and wheel. If $M^{*}$ is a cell corresponding to an orbit $O$ with $\operatorname{twn}(O)=m$, choose any normal map $F^{*}: M^{*} \rightarrow N$ with $F^{*}\left(\partial M^{*}\right)$ $=O$. Such maps exist $\left[8\right.$, p. 315] and $\mu\left(F^{*}\right)=m-1$. Define $F: M \rightarrow N$ by taking $F$ restricted to $M^{*}$ to be $f^{*}$ for each cell $M^{*}$ in $M$ corresponding to an orbit. As before, $\chi(M)=\zeta-\nu-\pi+\beta$. The multiplicity of $F$ can be determined using the following two observations, where the orbits of $A$ are $O_{1}, O_{2}, \ldots, O_{\zeta}$,

(i) $\operatorname{twn}(f \cup W)=\sum\left\{\operatorname{twn}\left(O_{i}\right) \mid i=1, \ldots, \zeta\right\}-\nu$.

Decompose $f \cup W$ into a set of regular closed curves approximating the orbits by cutting along the pairs and fans. Note that a cut along a pair increases the tangent winding number by one whereas a cut along a fan does not change the tangent winding number. The result follows since there are $\nu$ pairs,

(ii) $\operatorname{twn}(W)=\beta(\chi(N)-1)$.

Let $w$ be a wheel in $W$. and let $z_{1}, \ldots, z_{n}$ be the zeroes of the vector field with indices $s_{1}, \ldots, s_{n}$. We will observe that $\operatorname{twn}(w)=S-1$, where $S=\left\{\Sigma s_{i} \mid i=\right.$ $1, \ldots, n$ \}. First, if there are no zeroes, $S=0$ and (again by Lemma 2.6 of [3]) $\operatorname{twn}(w)=-1$. If there is only one zero, $z_{1}$, it follows directly from the definitions 
that $\operatorname{twn}(w)$ is one less than the index of $z_{1}$, that is, $\operatorname{twn}(w)=S-1$. If $n>1$, modify $w$ to obtain $w^{\prime}=w_{1} \cup w_{2}$ as shown in Figure 4, where $w_{1}$ bounds a disk containing only one zero, say $z_{1}$. Assuming the result holds for $n-1$ zeroes we have

$$
\begin{aligned}
\operatorname{twn}(w) & =\operatorname{twn}\left(w^{\prime}\right)+1 \\
& =\operatorname{twn}\left(w_{1}\right)+\operatorname{twn}\left(w_{2}\right)+1 \\
& =\left(S_{1}-1\right)+\left(S_{2}-1\right)+1, \quad \text { where } S_{2}=\left\{\Sigma s_{i} \mid i=2, \ldots, n\right\}, \\
& =S-1
\end{aligned}
$$

The observation follows by the induction principle. Since there are $\beta$ wheels in $W$, $\operatorname{twn}(W)=\beta(S-1)$. But by the Poincaré-Hopf index theorem [6, p. 134], $S=$ $\chi(N)$.

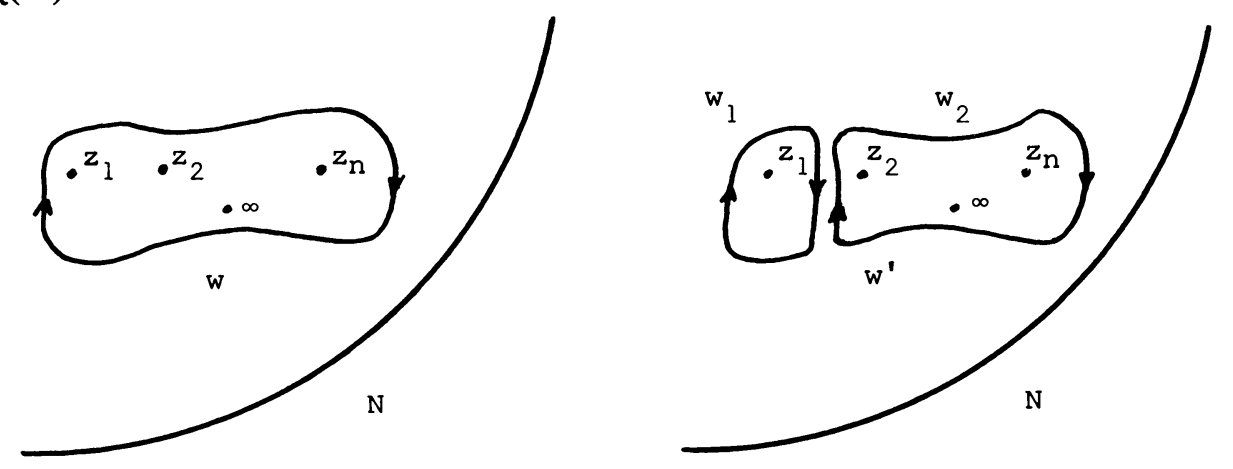

Figure 4

Now the multiplicity of $f$ can be calculated as follows:

$$
\begin{aligned}
\mu(f) & \left.=\pi+\sum\left\{\operatorname{twn}\left(O_{i}\right)-1 \mid i=1, \ldots, \zeta\right\} \quad \text { (by construction of } F\right) \\
& =\pi+\sum\left\{\operatorname{twn}\left(O_{i}\right) \mid i=1, \ldots, \zeta\right\}-\nu+\nu-\zeta \\
& =\pi+\operatorname{twn}(f \cup W)+\nu-\zeta \quad(\text { by }(\mathrm{i})) \\
& =\pi+\operatorname{twn}(f)+\operatorname{twn}(W)+\nu-\zeta \\
& =\pi+\operatorname{twn}(f)+\beta(\chi(N)-1)+\nu-\zeta \quad \text { (by.(ii)). } .
\end{aligned}
$$

Note that if the orbits of $A$ are all simple, then the construction above determines a unique (up to equivalence) map $F_{A}$, since $F_{A}$ restricted to each cell must be a homeomorphism. However, if the orbits are not all simple, then there is more than one way to define $F$ restricted to the cells, hence more than one way to construct an extension. Thus, a simple assemblage identifies a particular polymersion extension of a set of curves whereas an effective assemblage shows the existence of extensions.

Note 1. If $A$ is an assemblage on a set of curves $f$ in $N$ with orbits $O_{i}$, $i=1, \ldots, \zeta$, then from (i) and (ii) we have that $\operatorname{twn}(f)+\beta(\chi(N)-1)=$ $\Sigma\left\{\operatorname{twn}\left(O_{i}\right) \mid i=1, \ldots, \zeta\right\}$. The orbits are simple if and only if $\Sigma\left\{\operatorname{twn}\left(O_{i}\right) \mid i=\right.$ $1, \ldots, \zeta\}=\zeta$. Hence $A$ is simple if and only if $\nu+\operatorname{twn}(f)-\zeta+\beta(\chi(N)-1)=$ 0 . In the case where there are no wheels and $\beta$ is 0 , we see that simple assemblages are precisely the faithful assemblages of [4]. 
Note 2. Let $A$ be a transitive, effective assemblage on a set of curves $f$ in $N$. Any assemblage $\bar{A}$ obtained by adding more rays and extending $S$ and $P$ to the new crossings is called an extension of $A$. Suppose $A$ defines a map from a surface $M$ with characteristic $\chi$. An extension of $A$ which is simple and defines a map from a surface $M^{*}$ also of characteristic $\chi$, is called a maximal $\chi$-extension of $A$. (A maximal extension maximizes $\pi$ for fixed $\chi$.) It is easily seen that any assemblage (which may not be simple) has a maximal extension (which is simple) by choosing any map $F$ determined by $A$ and taking $\bar{A}$ to be the assemblage induced by $F$.

If a curve in a surface has a polymersion extension, then generally it is not difficult to determine this by finding an effective, transitive assemblage. However, finding a simple assemblage (which is more desirable since it defines a particular equivalence class of maps and locates all branch points) is generally harder. The following discussion will show how to obtain a maximal extension of any effective, transitive assemblage. Note the similarity of this treatment and that of Bailey [1] for planar $N$.

Let $f$ be a single curve in a surface $N$ having assemblage (with empty wheel set) $A=(R S P)$. Let $Q$ and $T$ be cycles of $P$. We say $Q$ and $P$ are linked if for some $q, q^{\prime} \in Q$ and $t, t^{\prime} \in T, S$ has the following form: $\left(A q B t C q^{\prime} D t^{\prime}\right)$, where the capital letters represent strings of letters in $S$. The following observation follows from the results in [8].

(iii) If $f$ is a single curve contained in a disk in a surface $N, R$ a full raying on $f$, and $S$ the successor permutation, and if $P$ is chosen so that it satisfies the following:

(a) $P$ is effective,

(b) the choice of $P$ maximizes $\pi$ subject to the condition that $P$ has no linked cycles,

(c) the order of the elements in the cycles of $P$ are the reverse of their order in $S$, then the assemblage $A=(R S P)$ is simple and describes a polymersion extension of $f$ to the disk.

Now let $f$ be a set of curves in a surface $N$ with a transitive effective assemblage $A=(R W S P)$ and with orbits $\left\{O_{i} \mid i=1, \ldots, \zeta\right\}$. The following steps will produce a maximal extension of $A$.

(A) Add rays $R^{*}$ so that the new raying $R^{\prime}=R \cup R^{*}$ is full on each orbit. This can be accomplished by choosing a saturated extension of $R$, i.e., select $R^{*}$ by taking one ray originating in each bounded component of $N-(f \cup W \cup R)$.

(B) Let $S^{\prime}$ be the successor permutation of $f$ relative to $R^{\prime}$, let $P^{*}$ be $P$ extended by the identity to the new crossings, and let $S_{1}, \ldots, S_{\zeta}$ be the cycles of $S^{\prime} P^{*}$ restricted to the new crossings. Then the permutations $S_{1}, \ldots, S_{\zeta}$ are successor permutations for the orbits $O_{1}, \ldots, O_{5}$. For each $i$, choose $P_{i}$ to be an assembling permutation for $O_{i}$ relative to the raying $R^{\prime}$ which satisfies (a), (b) and (c) of observation (iii). Hence by (iii), $\left(R^{\prime} S_{i} P_{i}\right)$ is an assemblage with simple orbits $O_{i, 1}, O_{i, 2}, \ldots, O_{i, n_{i}}$.

(C) Extend $P$ to $P^{\prime}$ by defining $P^{\prime}$ equal to $P_{i}, i=1, \ldots, n_{i}$, on the new crossings of $f \cup W$. The result is an assemblage $\left(R^{\prime} W S^{\prime} P^{\prime}\right)$ where each orbit $O_{i}$ 
has been replaced by simple orbits $O_{i, 1}, O_{i, 2}, \ldots, O_{i, n_{i}}$.

The following example illustrates these steps. For simplicity the saturated raying has not been used in this example.

2.4. EXAMPLE.

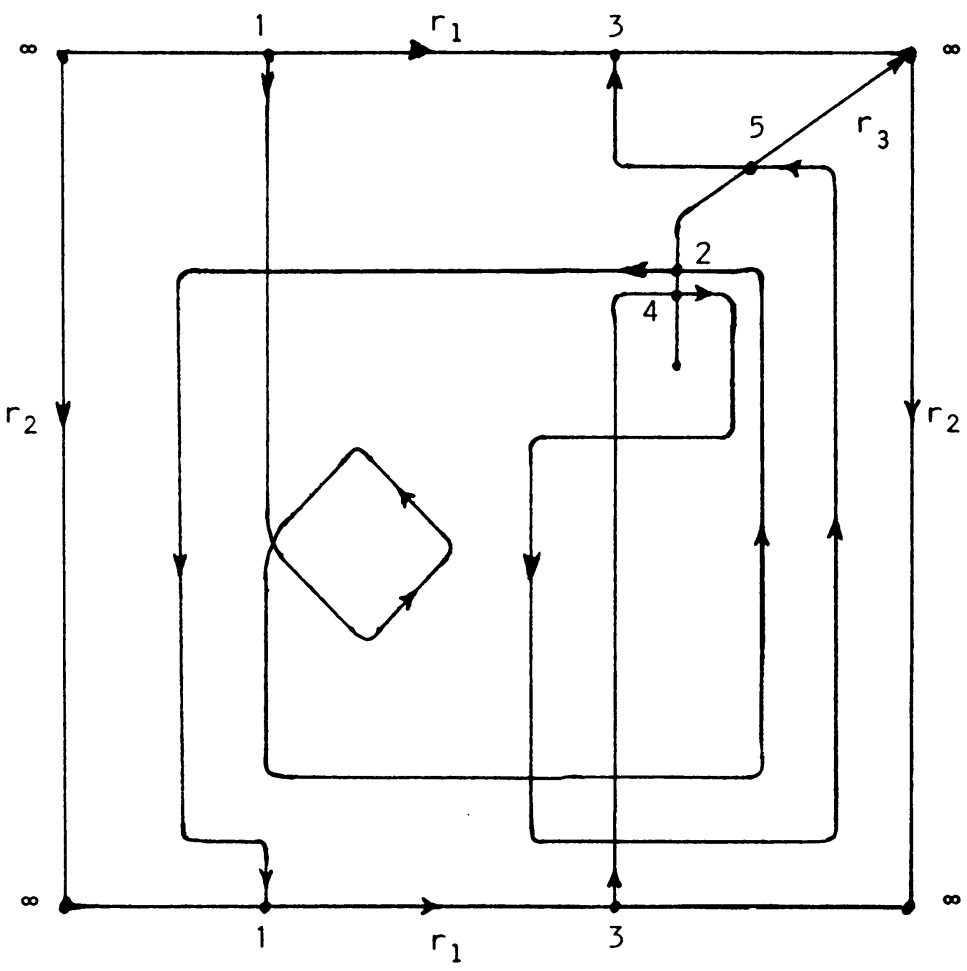

$$
\begin{array}{ll}
R=\left\{r_{1}, r_{2}, r_{3}\right\}, & W=\varnothing \\
S=\left(\begin{array}{ll}
1 & 2
\end{array}\right)(345), & P=\left(\begin{array}{ll}
1 & 3
\end{array}\right)(24)(5)
\end{array}
$$

Above is an example of an effective, transitive assemblage on a pair of curves in a torus. Its orbits are

$$
O_{1}=(1,2,4,5,3,1), \quad O_{2}=(3,4,2,1,3)
$$

This assemblage is not simple since $O_{1}$ cannot be approximated by a simple closed curve. ( $O_{1}$ contains transverse self-intersections.)

A maximal extension of this assemblage is illustrated below. 


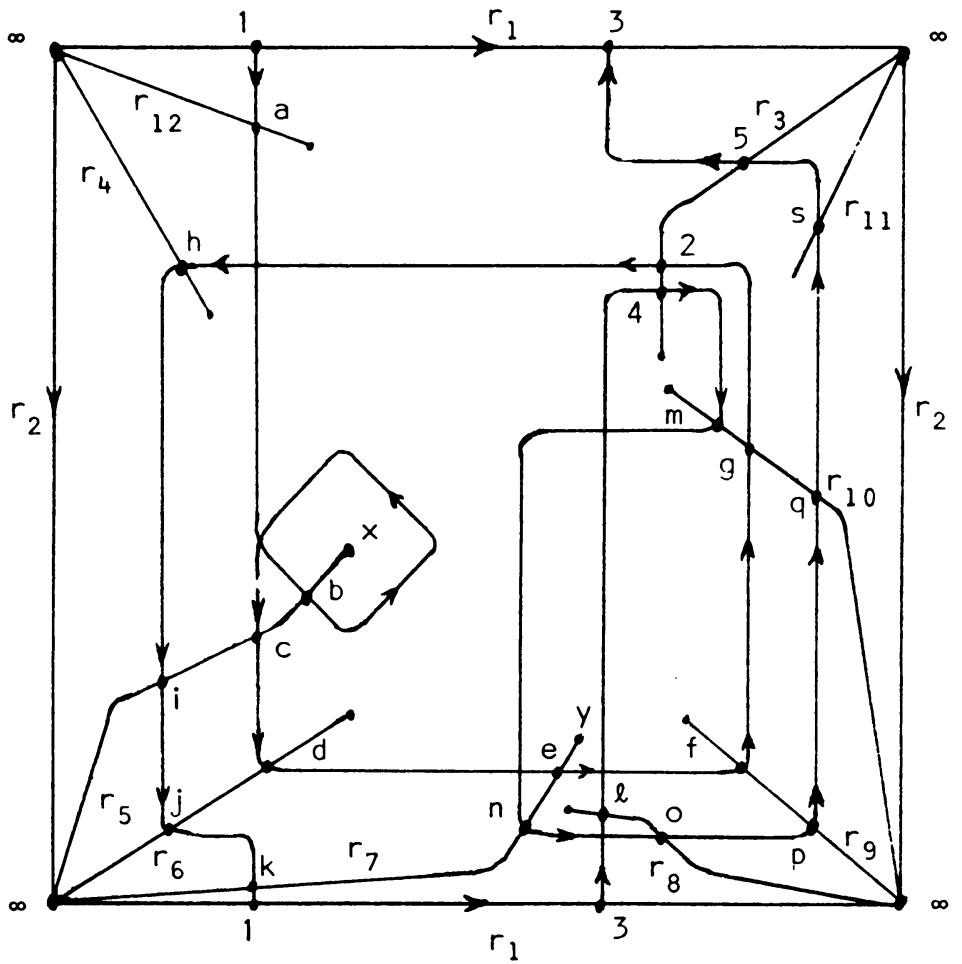

$$
\begin{aligned}
R^{\prime} & =\left\{r_{1}, r_{2}, r_{3}, r_{4}, r_{5}, r_{6}, r_{7}, r_{8}, r_{9}, r_{10}, r_{11}, r_{12}\right\}, \\
S^{\prime} & =(1 a b c d \text { efg } 2 h i j k)(3 l 4 m \text { mop } q s), \\
P^{*} & =(13)(24)(5)(I), \text { where }(I) \text { is the identity on crossings a through }, \\
S^{\prime} P^{*} & =(1 a b c d \text { efg } 4 m n \text { op } q s)(3 l 2 h i j k), \\
S_{1} & =(a b c d e f g m n o p q s), \\
S_{2} & =(l h i j k) .
\end{aligned}
$$

The crossings in $S_{1}$ that lie on the same ray are indicated by connecting lines in the figure below. Note that crossing $m$ is the only negative one.

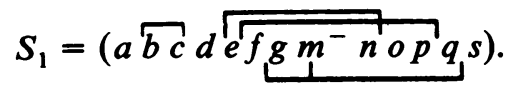

Hence, there are three ways of choosing pairs and fans without linking,

$$
\begin{gathered}
\left(a b c d e f g m^{-} n o p q s\right), \quad\left(a b c d e f \sqrt{g m^{-} n o p} q s\right), \\
(a b c d e f g \underbrace{m^{-}} \text {opp } q s) .
\end{gathered}
$$

The first two produce branching numbers of 2 and the third has branching number equal to 1 . Hence the first or second choice maximizes $\pi$. For this example we will choose the first and hence $P_{1}$ is as follows:

$$
P_{1}=(b c)(e n)(m g)(a)(d)(f)(o)(p)(q)(s) .
$$


Since all crossings in $S$ lie on different rays, there is only one choice for $\boldsymbol{P}_{2}$,

$$
P_{2}=(l)(h)(i)(j)(k) \text {. }
$$

Hence we have $P^{\prime}$ as follows:

$$
P^{\prime}=\left(\begin{array}{ll}
1 & 3
\end{array}\right)(24)(5)(b c)(e n)(m g)(a)(d)(f)(o)(p)(q)(s)(l)(h)(i)(j)(k) .
$$

Note that orbit $O_{1}$ has been replaced by the simple orbits

$$
\begin{aligned}
& O_{11}=(1, a, b, x, c, d, e, y, n, o, p, q, s, 5,3,1), \\
& O_{12}=(c, x, b, c), \\
& O_{13}=(4, m, n, y, e, f, g, 2,4) .
\end{aligned}
$$

Orbit $\mathrm{O}_{2}$ was simple and is unchanged in the extension.

3. Extensions of normal curves. The results of the previous section will now be used to establish some theorems on the existence of extensions of normal curves.

First we relate GPC to standard notions in algebraic and differential topology. Following [6, p. 107] we shall use $I(f, \gamma)$ to denote the oriented intersection number of the curve $f$ on an orientable surface $N$ and a cycle $\gamma$ on $N$. In this notation, GPC becomes $I\left(f, \gamma_{i}\right)=0,1 \leqslant i \leqslant k$, where $\gamma_{1}, \gamma_{2}, \ldots, \gamma_{k}$ are cycles representing a minimal generating set for the fundamental group of $N$.

3.1. TheOREM. The following are equivalent for a family of regular curves $f$ on an oriented surface $N$.

(1) $f$ satisfies GPC.

(2) $I(f, \gamma)=0$ for every cycle $\gamma$ on $N$.

(3) $f$ is null homologous.

Proof. It is easy to see that $I(f, \cdot)$ is a linear function on the elements of $Z_{1}(N)$, so (1) implies (2). A fortiori, (2) implies (1). If $f$ is closely approximated by a piecewise linear family $f^{*}$, the Lefschetz theory of the intersection of chains applies. His Theorem I of [7, p. 178] then gives the equivalence of (2) and (3).

We now wish to show that the conditions above are also equivalent to the existence of polymersion extensions of $f$ to some bordered surface $M$. If such an extension exists, it induces a complex analytic structure on $M$. By the first proposition on p. 108 in [6], we obtain (2). Before we show the converse, we make a definition.

Definition. Suppose $f$ is a family of regular curves satisfying (1), (2) or (3) above. Pick a point $\infty$ in $N$ but not on [f]. Let $x$ be any other point on $N$ but not on $[f]$. We define the winding number of $f$ at $p$ (with respect to $\infty$ ) by $\omega(f, p)=$ $I(f, r)$, where $r$ is any ray from $p$ to $\infty$ transverse to $f$. It follows immediately from (2) that this definition is independent of the choice of $r$. It is trivial to verify the constancy of $\omega(f, p)$ on components of $N-[f]$. From the properties of oriented intersection, we see the winding number is invariant under suitably restricted homotopies of $f$. Thus we have an analogue of the usual concept of winding number for planar curves.

Note. In the theorems to follow, we use the hypothesis of GPC. Obviously, in view of Theorem 3.1, either of the other two conditions is equally valid. 
3.2. THEOREM. Suppose $f$ is a family of regular curves satisfying GPC. If $F$ is any extension of $f$ to a bordered orientable surface, then

$$
\left|F^{-1}(x)\right|-\left|F^{-1}(\infty)\right|=\omega(f, x)
$$

for any $x \in N-[f]$. As is customary, when $x$ is a branch point, $\left|F^{-1}(x)\right|$ is calculated with regard to the multiplicities of the points in $F^{-1}(x)$.

Proof. Suppose an extension $F$ exists. Then $F$ induces an assemblage on $f \cup W$, where $W$ is a collection of $\left|F^{-1}(\infty)\right|$ wheels. Without loss of generality, we may assume the rays are chosen so as to have a ray $r$ with starting point $x$. There is a pairing on $r$, meaning that every lift of $r$ beginning at the preimage of a negative crossing terminates at a positive crossing, and the lift does not intersect $F^{-1}(x)$. On the other hand, every lift of the ray from the preimage of an unpaired positive crossing terminates at a point of $F^{-1}(x)$ and every lift of $r$ beginning at a point of $F^{-1}(x)$ terminates at the preimage of a paired positive crossing. From this we see that

$$
\begin{aligned}
\left|F^{-1}(x)\right|= & \text { number of unpaired positive crossings } \\
= & \text { number of positive crossings on } r \\
& - \text { number of negative crossings on } r .
\end{aligned}
$$

This last difference is just the definition of $\omega(f \cup W, x)$. The proof is completed by noting

$$
\omega(f \cup W, x)=\omega(f, x)+\omega(W, x)=\omega(f, x)+\left|F^{-1}(\infty)\right| .
$$

3.3. Corollary. Let $f, F$ be as above. Then

$$
\left|F^{-1}(\infty)\right| \geqslant \max _{x \in N-[f]}(-\omega(f, x)) .
$$

Thus, if $F^{-1}(\infty)=\varnothing$, then $\omega(f, x)>0$ for all $x \in N-[f]$.

Proof. From the theorem,

$$
\left|F^{-1}(\infty)\right|=\left|F^{-1}(x)\right|-\omega(f, x)>-\omega(f, x) .
$$

Next we demonstrate the existence of extensions achieving the lower bound above, and thereby obtain a generalization of the main result of [9].

3.4. Theorem. Suppose $f: K \rightarrow N$ is a normal mapping satisfying GPC. Let $\beta$ be any integer $\geqslant \max _{x \in N-[f]}(-\omega(f, x))$. If the curves in $f$ form a connected set then there exists a polymersion extension $F$ of $f$ with $\left|F^{-1}(\infty)\right|=\beta$. If $[f]$ is not connected, the same conclusion holds, but only for $\beta>\max (-\omega(f, x))$.

Proof. Let $W$ be a set of $\beta$ wheels; then by the hypothesis on $\beta, \omega(f \cup W, x) \geqslant$ 0 for all $x$. We show that this condition implies the existence of an effective pairing on any raying for $f$.

Suppose $r$ is a ray, as usual, transverse to $f$. Suppose $c_{1}, c_{2}, \ldots, c_{k}$ are the crossings on $r$, numbered so that $c_{i}$ precedes $c_{i+1}$ on $r, 1<i<k-1$. Pick some negative crossing, say $c_{j}$. Let $x$ be a point on $r$ between $c_{j-1}$ and $c_{j}$. The ray $r$ viewed only from $x$ to $\infty$ can be used to calculate $\omega(f \cup W, x)$, which is the sum 
of the signs of the $c_{i}, j \leqslant i \leqslant k$, and is $\geqslant 0$. Now suppose $c_{j}$ is the last (i.e., closest to $\infty$ ) negative crossing on $r$. By the preceding argument there is at least one positive crossing further out on $r$. Pair $c_{j}$ with the positive crossing closest to $\infty$. Now move back to the next negative crossing, say $c_{m}, m<j$. Then there are are at least two positive crossings beyond $c_{j}$. Pair $c_{m}$ with the unused positive crossing closest to $\infty$. Continue in this way to produce an effective pairing.

The existence of the desired extension would be immediate if we knew the above pairing to be transitive. However, if, for example, the curves in $f$ are disjoint, it will not be.

If $[f]$ is connected, we can choose the rays so that, for any pair of intersecting curves of $f$, the first two crossings on some ray are positive crossings-one from each curve. Then add these pairs of positive crossings to the assemblage and we have transitivity.

Next suppose $[f]$ is not connected. As before, we can produce an effective assemblage, but, because of the hypothesis on $\beta$, only using $\beta-1$ of the $\beta$ wheels. Choose a ray crossing all the wheels in points $d_{1}, d_{2}, \ldots, d_{\beta}$, and add the cycle $\left(d_{1} d_{2} \ldots d_{\beta}\right)$ to the assemblage. Take a component curve of $f$; if it has a negative crossing $a$, then there is a pair $(a b)$ in the assemblage. Replace (ab) with (ac), where $c$ is the positive crossing on the previously unused $\beta$ th wheel. If the curve has no negative crossings, select any positive crossing $a$ and add $(a c)$ to the assemblage, where $c$ is as previously described. Repeat this process for each component of $[f]$, and a transitive assemblage results.

The existence of $F$ follows from Theorem 2.3.

In the case where $N$ is the sphere, there is particular interest in whether an extension $F$ exists with $\left|F^{-1}(\infty)\right|=0$; i.e., whether an extension is (topologically) analytic or meromorphic. The next corollary settles this type of question in answering an analogue of the Loewner-Titus conjecture [12, p. 218] for a compact oriented target $N$.

3.5. Corollary. Suppose $f: K \rightarrow N$ is normal with [f] connected, satisfying GPC with $\omega(f, x) \geqslant 0$ for all $x \in N-[f]$. Then there exists a compact bordered oriented surface $M$ and a polymersion $F: M \rightarrow N-\{\infty\}$ with $F \mid \partial M=f$.

3.6. Corollary. Suppose $f: S^{1} \rightarrow N$ is normal with $[f]$ connected, satisfying GPC. Then there is a closed oriented surface $M$ containing $S^{1}$ as a separating cycle and $a$ branched cover $F: M \rightarrow M$ of degree $\max _{x \in N-[f]}(-\omega(f, x))+\max _{x \in N-[f]}(\omega(f, x))$ with $F \mid S^{1}=f$.

Proof. Use Theorem 3.4 to produce a polymersion $F_{1}: M_{1} \rightarrow N$ where $\partial M_{1}=$ $S^{1}, F_{1} \mid S^{1}=f$, and $\left|F_{1}^{-1}(\infty)\right|=\max (-\omega(f, x))$. Also from the theorem applied to the curve $-f$, i.e., $f$ with the sense reversed, we obtain a polymersion $F_{2}: M_{2} \rightarrow N$ with $\partial M_{2}=S^{1}, F_{2} \mid S^{1}=-f$, and $\left|F_{2}^{-1}(\infty)\right|=\max (-\omega(-f, x))=\max (\omega(f, x))$. Then form $M$ by gluing $M_{1}$ and $M_{2}$ along $S^{1}$ and define $F$ in the obvious way. Note, $F$ is a branched cover and its degree is $\left|F^{-1}(\infty)\right|=\left|F_{1}^{-1}(\infty)\right|+\left|F_{2}^{-1}(\infty)\right|$.

The following is a generalization of the Riemann-Hurwitz equation for maps into the plane. 
3.7. TheOREM. Let $F: M \rightarrow N$ be a polymersion from a bordered surface $M$ to $a$ smooth, oriented surface $N$ with a vector field vanishing only at a finite number of points in a neighborhood $U \subset N-F(\partial M)$ of some fixed point. Then $\chi(M)+\mu(F)$ $=\operatorname{twn}(F \mid \partial M)+\beta \cdot \chi(N)$, where $\beta=\left|F^{-1}(\infty)\right|$.

Proof. Let $A$ be the assemblage induced by $F$. Then from Theorem 2.3 we have

(i) $\chi\left(M_{A}\right)=\zeta-\nu-\pi+\beta$.

(ii) $\mu\left(F_{A}\right)=\nu+\operatorname{twn}(F \mid \partial M)-\zeta+\pi+\beta(\chi(N)-1)$.

(Since $A$ is simple, $\nu+\operatorname{twn}(F \mid \partial M)-\zeta+\beta(\chi(N)-1)=0$ and $\mu\left(F_{A}\right)=\pi$. However, line (ii) holds for all transitive, effective assemblages, hence also for simple assemblages.) Now add lines (i) and (ii) to obtain $\chi\left(M_{A}\right)+\mu\left(F_{A}\right)=\operatorname{twn}(F \mid \partial M)+$ $\beta \cdot \chi(N)$. The result follows since $F_{A}$ is equivalent to $F$.

NoTE. The above result is obtained by different means in [10].

\section{REFERENCES}

1. K. D. Bailey, Extending closed plane curves to immersions of a disc with $\boldsymbol{n}$ handles, Trans. Amer. Math. Soc. 206 (1975), 1-24.

2. S. J. Blank, Extending immersions of the circle, Dissertation, Brandeis University, 1967; cf. Poenaru, Exposé 342, Séminaire Bourbaki, 1967-1968, Benjamin, New York, 1969.

3. D. R. J. Chillingworth, Winding numbers on surfaces. I, Math. Ann. 196 (1972), 218-249.

4. G. K. Francis, Assembling compact Riemann surfaces with given boundary curves and branch points on the sphere, Illinois J. Math. 20 (1976), 198-217.

5. , Polymersions with nontrivial targets, Illinois J. Math. 22 (1978), 161-170.

6. V. Guillemin and A. Pollack, Differential topology, Prentice-Hall, Englewood Cliffs, N. J., 1974.

7. S. Lefshetz, Topology, Amer. Math. Soc. Colloq. Publ., no. 12, Amer. Math. Soc., Providence, R. I., 1930.

8. M. L. Marx, Extensions of normal immersions of $S^{1}$ onto $R^{2}$, Trans. Amer. Math. Soc. 187 (1974), 302-326.

9. M. L. Marx and R. Verhey, Interior and polynomial extensions of immersed circles, Proc. Amer. Math. Soc. 24 (1970), 41-49.

10. J. R. Quine, A global theorem for singularities of maps between oriented 2-manifolds, Trans. Amer. Math. Soc. 236 (1978), 307-314.

11. C. J. Titus, The combinatorial topology of analytic functions on the boundary of a disc, Acta Math. 106 (1961), 45-64.

12. Extensions through codimension one to sense preserving mappings, Ann. Inst. Fourier (Grenoble) 23 (1973), 215-227.

Department of Mathematics, Stetson University, Deland, Florida 32720

Department OF MATHEMATICS, UNIVERStTY OF OKLAHOMA, NORMAN, OKLAHOMa 73019 